www.ijpsonline.com

\title{
Role of Flash Glucose Monitoring System Combined With Insulin Pump in Blood Glucose Treatment of Patients with Type 2 Diabetes Mellitus
}

\author{
JINXIA WANG*, TING ZENG, LU CAI, PING LIU AND HONGHUA WEN \\ Second Affiliated Hospital of Nanjing Medical University, Nanjing 210000, Jiangsu Province, China
}

Liu et al.: Monitoring of Type 2 Diabetes Mellitus

\begin{abstract}
To explore the role of flash glucose monitoring system combined with insulin pump in the blood glucose treatment of patients with type 2 diabetes mellitus. Eighty patients with type 2 diabetes mellitus treated in our hospital from September 2019 to September 2020 were randomly divided into two groups $(n=40)$ using a random number table. Both groups received hypoglycemic treatment using an insulin pump. During treatment, blood glucose level was measured by collecting the fingertip blood of control group, while dynamic blood glucose monitoring was carried out for observation group using the flash glucose monitoring system. Blood glucose indices, time of controlling blood glucose to target level, insulin dosage, incidence rate of hypoglycemia, comfort score; mental state scores, sleep quality score and quality of life score were compared. After treatment, fasting blood glucose and $2 \mathrm{~h}$ postprandial blood glucose were lower, time of controlling blood glucose to target level was shorter and insulin dosage was smaller in observation group than those in control group $(p<0.05)$. Observation group had lower incidence rate of hypoglycemia, Self-rating Anxiety Scale, Self-rating Depression Scale and Pittsburgh Sleep Quality Index scores, as well as higher comfort and quality of life scores than those of control group $(p<0.05)$. In the intensive hypoglycemic treatment of patients with type 2 diabetes mellitus using an insulin pump, the application of flash glucose monitoring system can effectively control blood glucose, accelerate blood glucose control and reduce insulin dosage and hypoglycemia reaction, which is helpful to raise the comfort level of patients, ameliorate the psychological and sleep states and improve the quality of life.
\end{abstract}

Key words: Type 2 diabetes mellitus, hypoglycemic drug, flash glucose monitoring system, insulin pump

Type 2 diabetes mellitus, a common clinical disease characterized by "hyperglycemia", is a chronic metabolic disease, which mostly occurs in the elderly. The treatment method for type 2 diabetes mellitus is mainly hypoglycemic drug therapy ${ }^{[1-3]}$. Insulin pump, a commonly used method of insulin administration in treating patients with type 2 diabetes mellitus, can be used for continuous infusion of insulin to control the blood glucose level ${ }^{[4]}$. During treatment, to better control the blood glucose, it is clinically advocated to adjust the insulin dosage according to the real time blood glucose value ${ }^{[5]}$. Previously, blood glucose was determined through collection of fingertip blood for multiple times, showing operation inconvenience. In recent years, flash glucose monitoring system has been gradually used in clinical blood glucose management, the operation of which is more convenient. To investigate the effect of flash glucose monitoring system in insulin pump treatment of patients with type
2 diabetes mellitus, this study was conducted for 80 patients with type 2 diabetes mellitus who were treated in our hospital from September 2019 to September 2020. A total of 80 patients with type 2 diabetes mellitus who were treated in our hospital from September 2019 to September 2020 were enrolled and randomly divided into two groups $(\mathrm{n}=40)$ using a random number table. In control group, there were 21 males and 19 females aged $60-84 \mathrm{y}$ old, with an average of $(71.43 \pm 9.14) \mathrm{y}$ old and the disease course ranged 1-8 y, with a mean of $(4.85 \pm 1.42) \mathrm{y}$. In observation group, there were 22 males and 18 females aged $60-85 \mathrm{y}$ old, with an average of (71.68 \pm 9.32$) \mathrm{y}$ old and the disease course ranged 1-9 $\mathrm{y}$, with a mean of $(4.98 \pm 1.40)$ y. No significant differences were observed in the age, gender and course of disease between the two groups ( $>0.05)$, which were comparable. This study was approved by the hospital's ethics committee and all subjects signed the informed consent. Both groups received hypoglycemic 
treatment with an insulin pump. Specifically, Novolin $\mathrm{N}$ was loaded into the insulin pump, the needle was punctured through the lateral buttocks of the patient and the syringe needle was imbedded under the skin. The dosage was $0.5 \mathrm{U} / \mathrm{kg}$ per $\mathrm{d}$ initially and then adjusted once every $4 \mathrm{~d}$ according to the fasting blood glucose value of the patients. The patients were treated for 2 consecutive weeks. During treatment, the blood glucose was detected through collection of fingertip blood for multiple times in control group. Specifically, the patients' fingertip blood samples were collected in the early morning on an empty stomach, $2 \mathrm{~h}$ after breakfast, $2 \mathrm{~h}$ after lunch and $2 \mathrm{~h}$ after dinner. The blood glucose was detected using a Contour TS blood glucose meter (Bayer, Germany), 4 times a $\mathrm{d}$ and the results were recorded in the registration form. In observation group, the flash glucose monitoring system was used for dynamic blood glucose monitoring. Specifically, the sensor of the Freestyle Libre Flash Glucose Monitoring System (Abbott Laboratories, USA) was pasted on the upper back of the patient's arm, blood glucose measurement could be completed by pressing the scanner in the early morning on an empty stomach, before meals, after meals and before going to bed and the data were automatically uploaded to the software system after scanning. Indices of blood glucose (fasting blood glucose and $2 \mathrm{~h}$ postprandial blood glucose), time of controlling blood glucose to target level (when fasting blood glucose $<7.0 \mathrm{mmol} / 1$, blood glucose was under control), insulin dosage, incidence rate of hypoglycemia, comfort score, mental state scores, sleep quality score and quality of life score were compared between the two groups. Comfort score ${ }^{[6]}$ : The General Comfort Questionnaire (GCQ) was used to evaluate the comfort of patients, which included a total of 28 items, with 1-4 points for each item and the total score was 28-112 points. The higher the score, the better. Mental state scores ${ }^{[7]}$ : Self rating Anxiety Scale (SAS) was employed to evaluate anxiety, with a total score of 100 points and the critical value was 50 points. The higher the score, the more anxious the patient. Self-rating Depression Scale (SDS) was used to assess depression, with a total score of 100 points and the critical value was 53 points. The higher the score, the more depressed patient. Sleep quality score $^{[8]}$ : Pittsburgh Sleep Quality Index (PSQI), comprising 7 items (0-3 points/item, $0-21$ points in total), was selected for sleep quality evaluation. The higher the score, the more serious the sleep quality disorder. Quality of life score ${ }^{[9]}$ : The World Health Organization Quality of Life Instrument, Short Form (WHOQOL-BREF) (formulated by the World Health Organization) was used to evaluate the quality of life of patients. The scale involves physiology, psychology, environment and social relations (0-100 points for each part). The higher the score, the better. SPSS 22.0 software was utilized for statistical analysis. The $\chi^{2}$ test was performed for numerical data (n) and the t-test was conducted for quantitative data $(\chi \pm s) . p<0.05$ suggested that the difference was statistically significant. Compared with those before treatment, fasting blood glucose and $2 \mathrm{~h}$ postprandial blood glucose declined significantly in both groups after treatment $(\mathrm{p}<0.05)$ and they were lower in observation group than those in control group after treatment $(\mathrm{p}<0.05)$ (Table 1). Time of controlling blood glucose to target level was shorter in observation group than that in control group $(p<0.05)$, while insulin dosage was smaller in observation group than that in control group $(\mathrm{p}<0.05)$. Besides, observation group had a lower incidence rate of hypoglycemia than control group $(\mathrm{p}<0.05)$ (Table 2). Compared with those before treatment, SAS and SDS scores dropped significantly in both groups after treatment $(\mathrm{p}<0.05)$ and observation group had significantly lower SAS and SDS scores than control group after treatment $(p<0.05)$ (Table 3). In comparison with that before treatment, GCQ score rose significantly in both groups after treatment $(p<0.05)$ and observation group had a higher GCQ score than control group after treatment $(\mathrm{p}<0.05)$. Compared with that before treatment, PSQI score declined in both groups after treatment $(p<0.05)$ and observation group showed a lower PSQI score than control group after treatment $(\mathrm{p}<0.05)$ (Table 4$)$. Compared with that before treatment, quality of life score rose in both groups after treatment $(p<0.05)$ and observation group exhibited a higher quality of life score than control group after treatment $(p<0.05)$ (Table 5). Type 2 diabetes mellitus is a kind of chronic

TABLE 1: BLOOD GLUCOSE INDICES ( $\overline{\mathbf{x} \pm s, \mathrm{MMOL} / \mathrm{L})}$

\begin{tabular}{|c|c|c|c|}
\hline Group & Time & Fasting blood glucose & 2-h postprandial blood glucose \\
\hline \multirow{2}{*}{ Control $(n=40)$} & Before treatment & $9.12 \pm 1.54$ & $12.74 \pm 2.08$ \\
\hline & After treatment & $7.58 \pm 1.19^{\#}$ & $10.69 \pm 1.65^{\#}$ \\
\hline \multirow{2}{*}{ Observation $(n=40)$} & Before treatment & $9.08 \pm 1.52$ & $12.67 \pm 2.10$ \\
\hline & After treatment & $5.45 \pm 1.03^{\# *}$ & $8.56 \pm 1.27^{\# *}$ \\
\hline
\end{tabular}

${ }^{\#} \mathrm{p}<0.05$ vs. before treatment, ${ }^{*} \mathrm{p}<0.05$ vs. control group. 
TABLE 2: TIME OF CONTROLLING BLOOD GLUCOSE TO TARGET LEVEL, INSULIN DOSAGE AND INCIDENCE RATE OF HYPOGLYCEMIA

\begin{tabular}{lcccc}
\hline Group & $\mathbf{n}$ & Time of blood glucose control $(\mathbf{d})$ & Insulin dosage $(\mathrm{U})$ & Incidence rate of hypoglycemia \\
\hline Control & 40 & $5.69 \pm 1.68$ & $54.37 \pm 9.85$ & $4(10.00 \%)$ \\
Observation & 40 & $4.03 \pm 1.25^{*}$ & $43.26 \pm 7.59^{*}$ & $0(0)^{*}$ \\
\hline
\end{tabular}

${ }^{*} \mathrm{p}<0.05$ vs. control group.

TABLE 3: MENTAL STATE SCORES ( $\overline{x \pm s}$, POINT)

\begin{tabular}{lccc}
\hline Group & Time & SAS score & SDS score \\
\hline \multirow{2}{*}{ Control $(n=40)$} & Before treatment & $54.39 \pm 6.86$ & $55.47 \pm 6.53$ \\
& After treatment & $47.10 \pm 5.27^{\#}$ & $48.59 \pm 5.42^{\#}$ \\
Observation $(n=40)$ & Before treatment & $54.21 \pm 6.90$ & $55.28 \pm 6.61$ \\
& After treatment & $40.74 \pm 4.93^{\# *}$ & $42.16 \pm 5.07^{\# *}$ \\
\hline
\end{tabular}

${ }^{\#} p<0.05$ vs. before treatment, ${ }^{*} p<0.05$ vs. control group.

TABLE 4: COMFORT AND SLEEP QUALITY SCORES ( $\overline{x \pm s}$, POINT)

\begin{tabular}{lccc}
\hline Group & Time & GCQ score & PSQI score \\
\hline \multirow{2}{*}{ Control $(n=40)$} & Before treatment & $74.62 \pm 7.51$ & $15.34 \pm 2.69$ \\
& After treatment & $84.24 \pm 8.35^{\#}$ & $12.65 \pm 2.24^{\#}$ \\
Observation $(n=40)$ & Before treatment & $74.89 \pm 7.46$ & $15.27 \pm 2.75$ \\
& After treatment & $95.12 \pm 9.64^{\# *}$ & $10.41 \pm 1.98^{\# *}$ \\
\hline
\end{tabular}

${ }^{\#} p<0.05$ vs. before treatment, ${ }^{*} p<0.05$ vs. control group.

TABLE 5: QUALITY OF LIFE SCORES (X̄̄s, POINT)

\begin{tabular}{cccccc}
\hline Group & Time & Physiology & Psychology & Environment & Social relations \\
\hline \multirow{2}{*}{ Control $(n=40)$} & Before treatment & $70.81 \pm 5.20$ & $70.23 \pm 5.14$ & $70.34 \pm 4.91$ & $70.58 \pm 5.09$ \\
& After treatment & $77.34 \pm 6.42^{\#}$ & $77.87 \pm 6.17^{\#}$ & $76.46 \pm 5.23^{\#}$ & $76.73 \pm 5.40^{\#}$ \\
Observation $(n=40)$ & Before treatment & $70.96 \pm 5.17$ & $70.45 \pm 5.13$ & $70.48 \pm 4.99$ & $70.20 \pm 5.16$ \\
& After treatment & $84.05 \pm 6.59^{\# *}$ & $84.39 \pm 6.28^{\# *}$ & $82.47 \pm 5.38^{\# *}$ & $83.62 \pm 5.71^{\# *}$ \\
\hline
\end{tabular}

${ }^{\#} \mathrm{p}<0.05$ vs. before treatment, ${ }^{*} \mathrm{p}<0.05$ vs. control group.

disease with a long disease course, which is relatively common in clinics. Its clinical manifestation is mainly abnormal elevation of blood glucose level. Patients with type 2 diabetes mellitus are often accompanied by symptoms such as thirst, polyuria and fatigue severely harming the physical and mental health of patients ${ }^{[10,11]}$. Type 2 diabetes mellitus is more common in the elderly. With the increase of the elderly population in recent years, the incidence rate of type 2 diabetes mellitus exhibits an upward trend, so it has become a public health problem endangering people's health ${ }^{[12]}$. In clinical practice, the pathogenesis of type 2 diabetes mellitus is not completely clear and there is still a lack of means to cure type 2 diabetes mellitus. Its treatment mainly aims to control blood glucose using hypoglycemic drugs dominated by insulin that is often continuously infused with an insulin pump. This can continuously supplement insulin for patients and enable the patient's body to gradually adapt to the supplement of exogenous insulin, so they can absorb insulin steadily and slowly, thus constantly stimulating islet cells, facilitating the self-repair of islet cells and improving the islet cells ${ }^{[13]}$. During insulin pump treatment, the effect of blood glucose level control is closely related to the insulin infusion dosage. To improve the hypoglycemic effect and reduce the risk of hypoglycemia, the insulin infusion dosage needs to be adjusted in time, but how to determine blood glucose is a vital problem to be solved ${ }^{[14]}$. Previously, blood glucose was determined in diabetic patients mainly through collection of fingertip blood from patients for multiple times and the insulin infusion dosage was acutely adjusted according to the results of blood glucose detection. Thus, the operation of this blood glucose monitoring method was relatively tedious and could not sensitively reflect the changes in blood glucose value ${ }^{[15]}$. In recent years, the dynamic blood glucose monitoring system has been gradually applied in blood glucose management and the Freestyle Libre Flash Glucose Monitoring System is a new type of dynamic blood glucose monitoring instrument launched by Abbott Laboratories. The operation is mainly as follows: The sensor is placed on the upper part of the patient's arm (fingertip blood collection not needed), the button of the scanner is gently pressed and then blood glucose measurement is completed by the sensor and the real time blood glucose data are obtained. The operation is relatively simple and it is convenient for 
patients to better understand the changes in their own blood glucose, manage their blood glucose more accurately and reduce the fluctuation of blood glucose $^{[16]}$. In this study, it was found that: After treatment, fasting blood glucose and $2 \mathrm{~h}$ postprandial blood glucose were lower $(p<0.05)$, time of blood glucose control was shorter $(\mathrm{p}<0.05)$ and insulin dosage was smaller $(p<0.05)$ in observation group than those in control group. Besides, observation group had a lower incidence rate of hypoglycemia than control group $(p<0.05)$. These results suggest that the flash glucose monitoring system can enhance the blood glucose control effect of patients with type 2 diabetes mellitus, accelerate the controlling of blood glucose to target level and avoid excessive decrease in blood glucose. After treatment, observation group had a higher comfort score and quality of life score than control group $(p<0.05)$. Moreover, the SAS and SDS scores and PSQI score were lower in observation group than those in control group $(\mathrm{p}<0.05)$. This is mainly because the flash glucose monitoring system requires no collection of fingertip blood from patients, which can avoid the pain caused by fingertip blood sampling. Meanwhile, the flash glucose monitoring system improves the effect of blood glucose control and is able to reduce the influence of the disease on patients' psychological status, sleep status and quality of life. To sum up, in the intensive hypoglycemic treatment of patients with type 2 diabetes mellitus using an insulin pump, the application of flash glucose monitoring system can effectively control blood glucose, accelerate the controlling of blood glucose to target level and reduce insulin dosage and hypoglycemia reaction, which is helpful to raise the comfort level of patients, ameliorate their psychological and sleep status and improve their quality of life.

\section{Conflict of interests:}

The authors declared no conflicts of interest. The authors declared no conflicts of interest.

\section{REFERENCES}

1. Kruger DF, Edelman SV, Hinnen DA, Parkin CG. Reference guide for integrating continuous glucose monitoring into clinical practice. Diabetes Educ 2019;45:3S-20S.

2. Slugocki M, Bialonczyk D, Özdener AE. A Review of Emerging Technologies in Diabetes Management for MultipleDose Insulin-Injecting Patients With Type 2 Diabetes Who Self-monitor Blood Glucose. J Pharm Technol 2019;35:69-81.

3. Arnold SV, Bhatt DL, Barsness GW, Beatty AL, Deedwania PC, Inzucchi SE, et al. Clinical management of stable coronary artery disease in patients with type 2 diabetes mellitus: A scientific statement from the American Heart Association. Circulation 2020;141:e779-806.

4. Kant R, Gilreath NA, Verma V. Management of type 2 diabetes mellitus in the elderly population with cardiovascular diseases. CTDD 2019;4:101-3.

5. Azhar A, Gillani SW, Mohiuddin G, Majeed RA. A systematic review on clinical implication of continuous glucose monitoring in diabetes management. J Pharm Bioallied Sci 2020;12:102-11.

6. Powell J, Nylen ES, Myers J, Karasik P, Moore H, Faselis C, et al. SAT-LB96 Chronic Kidney Disease Incidence and Cardiorespiratory Fitness Association in Patients with Diabetes and/or Hypertension. JES 2020;4:SAT-LB96.

7. Sun N, Lou P, Shang Y, Zhang P, Wang J, Chang G, et al. Prevalence and determinants of depressive and anxiety symptoms in adults with type 2 diabetes in China: a crosssectional study. BMJ Open 2016;6:e012540.

8. Lou P, Qin Y, Zhang P, Chen P, Zhang L, Chang G, et al. Association of sleep quality and quality of life in type 2 diabetes mellitus: a cross-sectional study in China Diabetes Res Clin Pract 2015;107:69-76.

9. Lin CY, Lee TY, Sun ZJ, Yang YC, Wu JS, Ou HT. Development of diabetes-specific quality of life module to be in conjunction with the World Health Organization quality of life scale brief version (WHOQOL-BREF). Health Qual Life Outcomes 2017; $15: 167$.

10. Olamoyegun MA, Ayodele AO, Christianah EA, Akinyele AT. Incidence and Determinants of Reported Hypoglycaemia among Patients with Type 2 Diabetes Mellitus in a Tertiary Health Institution in Nigeria. J Diabetes Mellit 2020;10:51-63.

11. Abe H, Shikuma J, Suwanai H, Sano K, Okumura T, Kan K, et al. Assessing hypoglycemia frequency using flash glucose monitoring in older Japanese patients with type 2 diabetes receiving oral hypoglycemic agents. Geriatr Gerontol Int 2019;19:1030-5.

12. Yamada M, Okada S, Oda H, Nakajima Y, Bastie CC, Kasai $\mathrm{Y}$, et al. Evaluation of the relationship between glycated hemoglobin A1c and mean glucose levels derived from the professional continuous flash glucose monitoring system. Endocr J 2020;67:531-6.

13. Venkatakrishnan K, Chiu HF, Wang CK. Popular functional foods and herbs for the management of type-2-diabetes mellitus: A comprehensive review with special reference to clinical trials and its proposed mechanism. J Funct Foods 2019;57:425-38.

14. Landau Z, Raz I, Wainstein J, Bar-Dayan Y, Cahn A. The role of insulin pump therapy for type 2 diabetes mellitus. Diabetes Metab Res Rev 2017;33:e2822.

15. Haak T, Hanaire H, Ajjan R, Hermanns N, Riveline JP, Rayman G. Flash glucose-sensing technology as a replacement for blood glucose monitoring for the management of insulintreated type 2 diabetes: a multicenter, open-label randomized controlled trial. Diabetes Ther 2017;8:55-73.

16. Caswell M, Frank J, Viggiani MT, Pardo S, Dunne N, WarchalWindham ME, Morin R. Accuracy and user performance evaluation of a blood glucose monitoring system. Diabetes Technol Ther 2015; 17:152-8.

This is an open access article distributed under the terms of the Creative Commons Attribution-NonCommercial-ShareAlike 3.0 License, which allows others to remix, tweak, and build upon the work non-commercially, as long as the author is credited and the new creations are licensed under the identical terms This article was originally published in a special issue,
"Clinical Research in Pharmaceutical and Biomedical
Sciences" Indian J Pharm Sci 2021:83(1)Spl issue1;102-105 